\title{
Study on dental scaling for patients with haemophilia
}

\section{Effectiveness in controlling haemorrhage after dental scaling in people with haemophilia by tranexamic acid mouthwash A. P. H. Lee, C. A. Boyle, G. F. Savidge and J. Fiske Br Dent J 2005; 198: 33-38}

\begin{abstract}
Aims
To compare the effectiveness of tranexamic acid mouthwash (TAMW) in controlling gingival haemorrhage after dental scaling with that of using factor replacement therapy (FRT) prior to dental scaling in people with haemophilia.

Design and setting

Double-blind cross-over randomised control trial. Dedicated hospital dental practice for patients with inherited bleeding disorders.

Method

Sixteen patients with haemophilia who required dental scaling participated in this pilot study. The experimental treatment regime (ETR) involved transfusing each patient with saline before scaling both quadrants on one side of the mouth followed by oral rinsing with TAMW four times daily for up to eight days. The control regime (CR) involved giving each patient FRT before scaling the opposite side of the mouth followed by use of a placebo TAMW. Each patient underwent both treatments in a randomised sequence. Both the operator and the patients were unaware of which were the ETR and CR episodes. On both occasions the patient kept a log book of the rinsing regime and any post-operative bleeding. Additionally, a structured post-treatment telephone interview was conducted to assess the effectiveness and the patient acceptability of the ETR.
\end{abstract}

\section{Results}

Thirteen patients completed the study. No statistically significant difference was found in gingival bleeding and mouthwashing frequencies between the ETR and the CR $(p>0.05)$. Five patients reported no gingival bleeding with either the ETR or the CR. No patient, using either regime, required extra FRT due to gingival haemorrhage. All subjects found the ETR acceptable and easy and reported feeling safe in using TAMW alone to control gingival bleeding after dental scaling.

\section{Conclusion}

TAMW use after dental scaling was as effective as using FRT beforehand in controlling gingival haemorrhage for people with haemophilia.

\section{IN BRIEF}

- A pilot study is small and lacks the power to recommend changes in clinical practice. A larger multi-centre study may be the solution.

- The results from this study promise the possibility of reducing the need of using factor replacement therapy on a minor traumatic procedure in people with haemophilia.

- It gives the patients more freedom in determining their own treatment;

- reserve factor for more severe bleeding situations, thus;

- avoiding the disadvantages of factor replacement therapy- high cost, development of factor antibodies and risks of blood product contamination.

\section{COMMENT}

This paper describes a cross-over, double blind trial of the use of an anti-fibrinolytic agent, tranexamic acid, used as a mouthwash to control post-scaling haemorrhage in patients with haemophilia. In the active treatment regime (ETR), the patients were infused with saline as a placebo factor replacement. The control group, (CTR), received similar dental treatment with factor replacement and a placebo mouthwash. On completion of the programme, five of the thirteen participants reported no problems with bleeding. There were no statistically significant differences between the ETC and CTR groups. All of the people found the mouthwash easy to use and only one participant found the taste unacceptable. There was a general feeling in all of the patients that they felt safe using the mouthwash for scaling in the knowledge that there was a safety back-up of factor replacement if needed.

There were logistic reasons for the small sample size related to the nature of the condition as well as travel to a regional centre; patients with haemophilia are usually treated in recognised centres of excellence and in some cases patients have to travel considerable distances. According to the article, the use of tranexamic acid mouthwash, in preference to factor replacement therapy, transfers the responsibility of the haemostatic management from the dentist to the patient. It also reduces the anxiety on the part of dentists in providing care.

From the results of this small study it would be unwise to recommend a change in clinical practice. However, within the limitations of a pilot investigation, the study did show that the use of tranexamic acid mouthwash after dental scaling is well tolerated by patients and would appear to be as effective in this clinical dental situation as using factor replacement therapy.

The design of this study, as a cross-over, meant that each patient, irrespective of their underlying coagulation problems, had dental treatment either using factor replacement and a placebo mouthwash or placebo factor replacement and tranexamic acid mouthwash. However, there is no mention of the extent of periodontal pathology in each patient and also each patient's level of factor XIII or factor IX in relation to post-operative events.

In theory, the possibility of arranging for some of these patients to be treated locally within the community with sufficient back up from a recognised centre would appear to be the direction that people should be developing. This would require more targeted continuing dental education programmes to improve the knowledge and training of the general dental practitioners or community dental officers in each local area, so that their oral and dental care was managed safely. However, the study does point to the potentially useful role for tranexamic acid, as means of providing effective control of minor bleeding problems.

A larger sample size, based on a multi-centre population of patients with a range of coagulation problems and identified periodontal pathology would be useful in testing the use of alternative agents further.

B. Harrington, Senior Lecturer/Consultant in General Practice, Dublin Dental School and Hospital doi: 10.1038/sj.bdj.4811952 